\title{
CF Carrier testing in a high risk population: Anxiety, risk perceptions, and reproductive plans of carrier by "non-carrier" couples
}

Nancy P. Callanan, $M S^{1}$, Brian J. Cheuvront, $P h D^{2}$, and James R. Sorenson, $P h D^{3}$

\begin{abstract}
Purpose: The risk perceptions, psychological status and reproductive plans of 52 carrier by "noncarrier" (mutation screen negative) couples is the subject of this report. Methods: Cystic fibrosis (CF) carrier testing was offered to relatives of individuals with CF. Results: In this population testing was not associated with any significant adverse psychological effects, reproductive uncertainty, or inaccurate risk perceptions. Conclusions: The results of this study have important implications in light of the recent NIH CF Consensus Panel recommendations that CF carrier testing be offered to all high risk adults and all couples planning a pregnancy or seeking prenatal testing. Genetics
\end{abstract} in Medicine, 1999:1(7):323-327.

Key Words: Cystic fibrosis, carrier testing, reproductive uncertainty, risk perceptions, genetic counseling

In response to various concerns about the implementation of population-based carrier testing for cystic fibrosis (CF), the Ethical Legal and Social Implications (ELSI) Branch of the National Human Genome Research Institute funded seven CF pilot projects in 1991. The issues of test sensitivity and the potential that testing might result in confusion and reproductive uncertainty, especially among couples in which one member tests positive and the other has a negative mutation screen, were among some of the critical areas of concern. The purposes of the pilot projects were to assess interest in testing, develop effective methods for providing counseling, education and informed consent for testing, evaluate the influence of test results on reproductive behavior, and document the occurrence of psychological and social effects of testing.

In April 1997, a 14-member Consensus Panel was convened by the National Institutes of Health to assess the optimal practices for genetic testing for CF. In compiling their recommendations, the panel considered the data gathered by the pilot projects as well as other published reports. The panel recommended that CF carrier testing be offered to adults with a family history of $\mathrm{CF}$, couples currently planning a pregnancy, and couples seeking prenatal testing. ${ }^{1}$ The consensus conference report emphasized the need for testing to be accompanied by appropriate education, counseling, and informed consent so that individuals can make informed autonomous decisions re-

From the 'Department of Biologl, Lniversity of North Carolina at Givenstore. Circenshore. North Carolina: "J. Metropolitan Collegc. Buston L'mervite; Buston, Mussachusetts; and ${ }^{3}$ Department of Health Behavior and Health Eetucation, Sichool of Pullic Health, L'mersit!' of North Carolina at Chapd Hill, Chapel Hill. North Corrolima

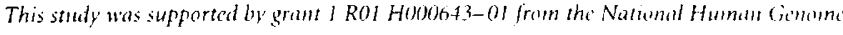
Rescarch Institute (previously known as the National Couter fur Humbn (ichome Rescarch).

Nancy P. Callanan, MS, Genetic Counseling Program, University of North Carolina at Greensboro, P.O. Box 26174, Greensboro. NC'27402-6174.

Recicived: September 1, 1999

Accepted: Noveniber 18, 1999. garding testing. Specifically, the report states that individuals to whom testing is offered should receive adequate and balanced information regarding the range of severity of the disease as well as the complex concepts of test sensitivity and risk. Presently a Steering Committee composed of representatives from the American College of Obstetrics and Gynecology, the American College of Medical Genetics, and the Ethical, Legal, and Social Implications Branch of the NHGRI is preparing professional practice guidelines for prenatal CF carrier testing. It is anticipated that these guidelines will be issued within the next 6 to 12 months.

In our ELSI funded CF Pilot Project, we offered cystic fibrosis carrier testing to relatives of individuals with CF. A total of 296 relatives were tested as part of this study and 120 CF carriers were identified. CF carrier testing was offered to the spouse or partner of all identified carriers, and genetic counseling was offered to all carriers regardless of their partner's decision to be tested. 57 partners were tested and 5 carriers were identified. The 5 carrier by carrier couples all received genetic counseling and were excluded from further participation in the study. The risk perceptions, psychological status and reproductive plans of the remaining 52 carrier by "noncarrier" couples are the subject of this report.

\section{MATERIALS AND METHODS}

\section{Subject Recruitment}

This study was approved by the University of North Carolina Institutional Review Board. Details of study participation have been reported previously ${ }^{2-4}$ and are summarized in Tables 1 and 2. A total of $320 \mathrm{CF}$ patients followed at the University of North Carolina CF Center were identified. Each patient, or their parents if the patient was a minor, was sent a letter from the CF Center Director describing the study. They were then contacted by telephone by a study researcher and asked to 
Table 1

Summary of patient recruitment

\begin{tabular}{lll}
\hline & $\begin{array}{c}\text { Clinic screen } \\
\mathrm{N}=163 \\
(50.9 \%)\end{array}$ & $\begin{array}{c}\text { Home screen } \\
\mathrm{N}=157 \\
(49.9 \%)\end{array}$ \\
\hline $\begin{array}{l}\text { Not eligible } \\
\begin{array}{l}\text { Not able to } \quad \text { contact } \\
\text { Eligible } \\
\text { Declined }\end{array}\end{array}$ & 28 & 21 \\
$\quad$ Passive refusal & 41 & 27 \\
Participated & 94 & 109 \\
\hline
\end{tabular}

"Passive refusal refers to individuals who agreed to participate but did not complete the process by providing contact information for their relatives.

Table 2

Summary of relative's participation

\begin{tabular}{|c|c|c|}
\hline & $\begin{array}{l}\text { Clinic screen } \\
\mathrm{N}=265\end{array}$ & $\begin{array}{l}\text { Home screen } \\
\mathrm{N}=434\end{array}$ \\
\hline Contacted & 205 & 309 \\
\hline Declined & 85 & 57 \\
\hline Accepted & 120 & 252 \\
\hline Passive refusal ${ }^{a}$ & 30 & 46 \\
\hline Tested & 90 & 206 \\
\hline
\end{tabular}

"Passive refusal refers to individuals who agreed to participate but did not complete the process by either scheduling an appointment for education and testing (Clinic Screen group) or submitting a sample for testing (Home Screen group).

participate by providing the names, addresses, and telephone numbers of their close relatives. Patients were also asked to submit a blood sample for CF mutation analysis if this had not been performed previously. The patients were randomly assigned to one of two testing arrangements: A clinic screen group in which relatives would receive traditional pretest education in clinic, and a home screen group, in which relatives would receive their pretest education by a specially designed educational brochure and would receive a saliva sample collection kit for mutation testing.

Our research participants included the siblings, aunts, uncles, and first cousins of individuals with CF in which the mutation status was known and carrier testing by direct mutation analysis was possible. Participants were initially contacted by letter with a follow up telephone contact. All participants were at least 18 years of age, not currently pregnant, and living in the study inclusion area (NC, SC, TN, VA, or WV).

\section{Education and Testing}

All education, counseling, and testing was provided at no cost to the participants. Individuals in the clinic screen group received pretest education/genetic counseling in clinic by a certified genetic counselor prior to testing. Individuals in the home screen group were educated by a specially designed ed- ucational brochure. The topics covered in the brochure matched topics generally covered in a pretest genetic counseling session including information about $\mathrm{CF}$, explanation of autosomal recessive inheritance, estimate of carrier risks for relatives, implications of being a $\mathrm{CF}$ gene mutation carrier, and information about how the testing is accomplished. The brochure was written at an eighth grade reading level and was pilot tested.

Carrier testing was performed on saliva samples by a reverse dot blot procedure. All subjects were screened for six common CF mutations (delta F508, G5513, G542X, R553X, W1282X, and $\mathrm{N} 1303 \mathrm{~K}$ ).

$\mathrm{CF}$ carrier testing was offered to the spouse or partner of any relative who was found to be a $\mathrm{CF}$ gene mutation carrier. Those who elected to be tested were screened for the above-mentioned six common CF mutations. All carriers were offered the opportunity to return for additional genetic counseling regardless of their partner's decision to be tested. Participants were informed of the limitations of testing at the time of the study, both verbally and in a results letter. We also recommended that they seek genetic counseling in the future if they had additional questions or concerns about their carrier status or test results.

\section{Psychological Measures}

Carrier relatives and their partners were surveyed during the interval between the partner's testing and receiving the results and 6 months later. The 10-item Spielberger State-Trait Anxiety Inventory (STAI) ${ }^{5,6}$ was used to measure anxiety as an emotional state and personality trait.

Positive and negative affect was measured by using the Positive and Negative Affect Scale (PANAS) developed by Watson and colleagues.? Positive affect is defined as "the extend to which a person feels enthusiastic, active and alert. High PA is a state of high energy, full concentration and pleasurable engagement, whereas low PA is characterized by sadness and lethargy." Negative affect (NA) is characterized as a general feeling of "distress and unpleasurable engagement..including anger, contempt, disgust, guilt, fear, and nervousness with a low NA being a stage of calmness and serenity."

\section{Risk Perceptions}

Prior to learning the partner's test results, carrier relatives and their partners were asked to give a numeric estimate of the partner's risk of being a CF gene mutation carrier and to evaluate this risk on a 5 point scale ranging from extremely high ${ }^{1}$ to extremely low. ${ }^{5}$ At the 6-month follow up, participants were also asked to give a verbal estimate of their children's risk for being a CF gene mutation carrier or for being clinically affected with $\mathrm{CF}$ using the same 5 point scale.

\section{Reproductive Plans}

The carrier relatives were asked about their reproductive plans upon entering the study and 6 months after learning their partner's carrier test results. The partners were also asked about their reproductive plans at the 6-month follow up. Al- 
though participants were not asked specifically to consider the role of CF in their reproductive plans, the questions were asked in this context.

\section{RESULTS}

\section{Education, Counseling, and Testing}

A total of 120 carriers were identified, 36 from the clinic screen group and 84 from the home screen group. $27 / 36$ individuals in the clinic screen group and 65/84 individuals in the home screen group had partners. Of these, $19(70.3 \%)$ of the clinic screen group partners and 38 (58.5\%) of the home screen group partners accepted the offer for $\mathrm{CF}$ carrier testing. Five $\mathrm{CF}$ gene mutation carriers were identified among the 57 partners tested. The five carrier by carrier couples received genetic counseling and were excluded from further participation in the study.

Few participants from either group returned for additional genetic counseling upon learning the results of their carrier testing. Results are summarized in Table 3.

\section{Psychological Measures}

The 10 item Spielberger State-Trait Anxiety Inventory (STAI) was used to measure participant anxiety. Spielberger reported that an average state anxiety score of the general public is $\mathbf{1 6 . 8 9}$ for males and 18.17 for females. ${ }^{5,6}$ Both relatives and their partners showed slightly higher anxiety scores while waiting for the partners test results, although the scores were not outside of the expected range for "normal" anxiety as measured by the STAI. There were no significant differences between the scores of the relatives and their partners at either point in time. Both the relatives and the partners showed a significant decrease in anxiety score 6 months after the completion of testing (Table 4).

Positive and negative affect were measured by the PANAS.? Carrier relatives scored in the average range for the general population while waiting for the partners' test results and six months later. There were no significant differences between relatives and their partners.

Table 3

Summary of testing and counseling

\begin{tabular}{lcl}
\hline & $\begin{array}{c}\text { Clinic } \\
(\mathrm{N}=90)\end{array}$ & $\begin{array}{c}\text { Home } \\
(\mathrm{N}=206)\end{array}$ \\
\hline Carrier & 36 & 84 \\
No partner & 9 & 19 \\
$\quad$ Partner & 27 & 65 \\
Partner tested & $19(70.3 \%)$ & $38(58.5 \%)$ \\
$\quad$ Carrier & 3 & 2 \\
$\quad$ Non-carrier & 16 & 36 \\
Counseling & $6(16.7 \%)$ & $11(13.1 \%)$ \\
\hline
\end{tabular}

Table 4

Change in anxiety scores over time

\begin{tabular}{lcc}
\hline & $\begin{array}{c}\text { Relatives } \\
(\mathrm{N}=30)\end{array}$ & $\begin{array}{c}\text { Partners } \\
(\mathrm{N}=32)\end{array}$ \\
\hline Waiting for results & 17.4 & 17.31 \\
Six months later & 14.7 & 15.0 \\
& $\mathrm{t}=3.16$ & $\mathrm{t}=3.05$ \\
& $p<0.01$ & $p<0.001$ \\
\hline
\end{tabular}

${ }^{a}$ Anxiety as measured by the Spielberger State-Trait Anxiety Inventory (STAI). General population average scores are 16.89 for men and 18.17 for women.

\section{Risk Perceptions}

Prior to learning the results of the partners' carrier testing, participants were asked to give an estimate of the partner's risk for being a CF gene mutation carrier and to rate this risk on a five point scale ranging from extremely low ${ }^{5}$ to extremely high. ${ }^{1}$ Relatives generally rated this risk to be low. When the responses of individual couples were analyzed using a paired comparison $t$ test, the partners' verbal estimates of their carrier risk was greater than that of the relative's estimate $(t=2.10$, $P<0.05$ ).

At the 6-month follow up relatives and their partners were asked to give a verbal estimate of their children's risk for being a CF gene mutation carrier or for being clinically affected with CF. Participants were asked to rate this risk on a five point scale ranging from extremely high ${ }^{1}$ to extremely low. ${ }^{5}$ As shown in Figure 1, participants estimated that their children's risk for being clinically affected with CF was "low" (3.7-4.0 on a 5 point scale), whereas their risk for being a carrier was "medium" (3) to "high" (2). These risk estimates suggest that participants understood that although their children's risk for being clinically affect was low, it was not zero, and that their children's risk for inheriting a CF gene mutation from their carrier parent was increased. There were no significant differences between the relatives' and partners' responses on these questions.

\section{Reproductive Plans}

Relatives and partners were asked about their reproductive plans at the 6-month follow up. More than $80 \%$ of couples were in agreement about their reproductive plans. In $48 \%$ of couples, both members of the couple stated that they did not plan on having a child in the future. $26 \%$ of couples were planning a child, and $7.4 \%$ of couples were not sure about their plans. Data are summarized in Table 5.

Relatives were also asked about their reproductive plans when they entered the study, prior to testing. We compared the responses of the relatives prior to testing with their responses after learning their test results and their partner's test results. The results are shown in Table 6. Approximately $89 \%$ of the relatives showed no change in reproductive plans after testing. At the 6-month follow up, $2.1 \%$ of the relatives who initially indicated that they were not planning additional children reported that they were planning additional children. Similarly, of relatives who initially reported that they did plan to have 


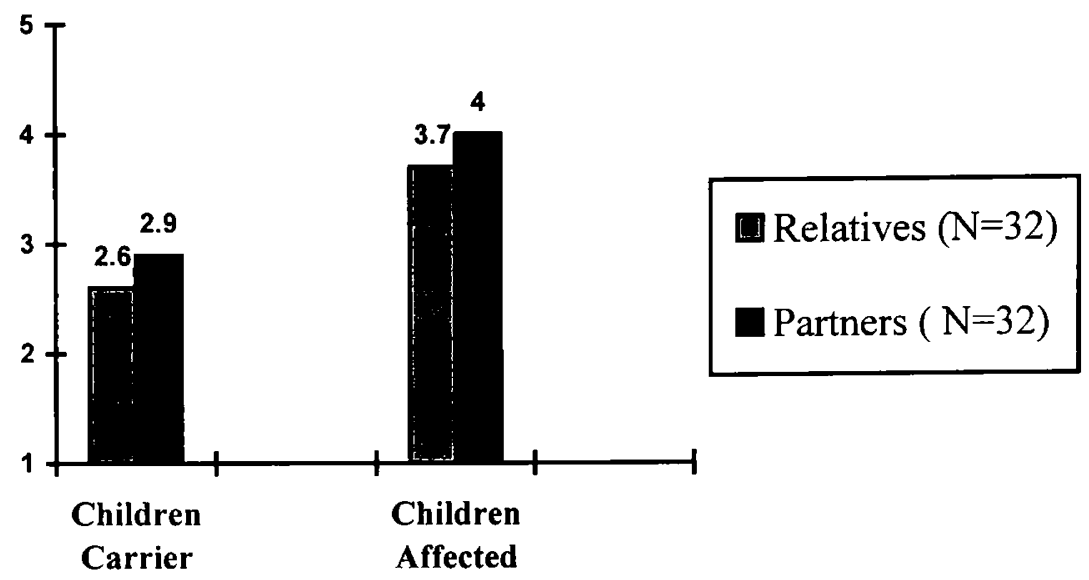

Fig. 1 Participants were asked to estimate the risk that children would be CF gene mutation carrier or clinically affected with CF on a 5 point scale ranging from extremely high (1) to extremely low (5).

Table 5

Reproductive plans of relatives and partners ${ }^{a}$

\begin{tabular}{lccc}
\hline $\begin{array}{l}\text { Partners } \\
(\mathrm{N}=32)\end{array}$ & \multicolumn{3}{c}{ Relatives $(\mathrm{N}=32)$} \\
\hline & No & Not Sure & Yes \\
No & $\mathbf{4 8 . 0 \%}$ & $3.7 \%$ & $0.0 \%$ \\
Not sure & $3.7 \%$ & $\mathbf{7 . 4 \%}$ & $11.1 \%$ \\
Yes & $0.0 \%$ & $0.0 \%$ & $\mathbf{2 6 . 0 \%}$ \\
\hline
\end{tabular}

"Participants' responses to the question "Do you plan to have a child in the future?"

Table 6

Reproductive plans of relatives ${ }^{a}$

\begin{tabular}{lccc}
\hline $\begin{array}{l}\text { Six months after } \\
\text { partner results }\end{array}$ & \multicolumn{3}{c}{ Baseline } \\
\hline & No & Not sure & Yes \\
No & $\mathbf{4 1 . 2 \%}$ & $0.0 \%$ & $2.1 \%$ \\
Not sure & $2.1 \%$ & $\mathbf{8 . 3} \%$ & $2.1 \%$ \\
Yes & $2.1 \%$ & $2.1 \%$ & $39.6 \%$ \\
\hline
\end{tabular}

a Relatives' response to the question, "Do you plan to have a child in the future?" upon entering the study (baseline) and 6 months after receiving the partners' test results.

children in the future, $2.1 \%$ reported that they did not plan additional children and $2.1 \%$ were not sure about future children.

\section{CONCLUSIONS}

In our population of 52 nonpregnant CF carriers (all of whom had a positive family history of $\mathrm{CF}$ ) and their partners, $\mathrm{CF}$ carrier testing was not associated with any significant increase in negative affect or anxiety. There was a slight increase in anxiety in both relatives and partners while waiting for the results of the partners' carrier testing, but within the range that is considered normal for the general population. By the 6-month follow up, there was a significant decrease in anxiety scores for both groups. These findings are similar to published reports that suggest that in the general population, CF carrier testing was associated with a short term increase in anxiety, but no long term negative psychological effects. ${ }^{1}$

Although none of the partners had genetic counseling prior to testing, and few couples returned for genetic counseling after the partner's testing was completed, the majority of our participants could provide an accurate estimate of their children's risk for being a CF gene mutation carrier or for being clinically affected with CF. For the partners, information about $\mathrm{CF}$ and $\mathrm{CF}$ carrier testing was transmitted via their partner who was either educated in clinic $(\mathrm{N}=19)$ or by a specially designed educational brochure at home $(\mathrm{N}=38)$. This suggests that pretest education of the relatives either in clinic or by the brochure was sufficient in providing information about $\mathrm{CF}$ carrier testing that the relative could accurately relay to his or her partner. Specifically, our participants seemed to understand that the child of a known CF gene mutation carrier has an increased risk for being a carrier and that for a couple in which one partner is a known CF gene mutation carrier and the other has a negative mutation screen, the risk for having a child clinically affected with CF is low.

Approximately $89 \%$ of relatives reported no change in their reproductive plans after testing. More than $80 \%$ of the couples agreed on their future reproductive plans, and $26 \%$ of couples intend to have children in the future. In only $11 \%$ of couples did one or both partners report that they were unsure about their future reproductive plans. It seems, therefore, that for our sample of carrier by "noncarrier" couples, CF carrier testing was not associated with significant reproductive uncertainty.

This study had several limitations when considering application to nonresearch situations. All education and testing was performed at no charge, and subjects had assurance of the confidentiality of their test results. Furthermore, the study protocol excluded pregnant individuals. In a clinical setting where individuals or couples are seeking testing motivated by their own desire to learn their carrier status and reproductive risk, it 
is likely that a higher proportion of partners of identified carriers would seek testing. It is also possible that testing performed in the context of a pregnancy may be associated with more significant anxiety.

We did not anticipate that that so few participants would return for follow up genetic counseling upon learning their carrier status. For most subjects in the Home Screen group, therefore, all education and testing was performed without any direct contact with a genetic counselor. Although this seemed adequate in terms of transmitting basic knowledge and risk information in this research setting, in a clinical setting, where individuals seek counseling and testing on their own accord, especially in the context of a pregnancy, it will be important to ensure the availability of follow up genetic counseling for identified carriers and their partners.

Our study focused on education, counseling, and testing for nonpregnant individuals with a positive family history of CF. Our results suggest that in this population, pretest education can be accomplished effectively with written materials. The NIH CF Consensus panel has recommended that in addition to offering CF carrier testing to any individual with a positive family history of CF, testing be offered to all couples contemplating a pregnancy and all couples seeking prenatal testing. It is likely that many of these individuals will have little or no prior experience with CF and will, therefore, have somewhat different informational needs prior to and after testing. None- theless, our experience with testing in this population of individuals at increased risk for $\mathrm{CF}$ suggests that couples in which one partner is identified as a carrier and the other partner has a negative mutation screen are, for the most part, able to comprehend the limitations of the testing and draw appropriate conclusions regarding the genetic implications of their test results.

\section{References}

1. NIH Consensus Development Conference Statement. Genetic testing for cystic fibrosis. Available at: http://odp.od.nih.gov/consensus/cons/106/106 statement.htm. Accessed August 10, 1999.

2. Cheuvront B, Sorenson J, Callanan N, Sterns S, DeVellis B. Psychosocial outcomes and costs associated with home and clinic-based pretest education and cystic fibrosis carrier testing among a population of at-risk relatives. Am J Med Genet 1998;75:461468.

3. Sorenson J, Cheuvront B, DeVellis B, Callanan N, Silverman L, Koch G, Sharp T, Fernald $G$. Acceptance of home and clinic based cystic fibrosis carrier education and testing by first, second, and third degree relatives of CF patients. Am J Med Genet 1997;70:121-129.

4. Sorenson YR, Cheuvront B, Bruning A, Talton S, DeVellis B, Koch G, Callanan N, Fernald $G$. Proband and parent assistance in identifying relatives for cystic fibrosis carrier testing. Am J Med Genet 1996;63:419-425.

5. Spielberger CD. Preliminary Manual for the State-Trait Personality Inventory. Tampa: Human Resources Institute, University of South Florida, 1979;

6. Spielberger CD. Assessment of state and trait anxiety: Conceptual and methodological issues. South Psychologist 1985;2:6-16.

7. Watson D, Clark LA, Tellegen A. Development and validation of a brief measure of positive and negative affect: The PANAS scales. J Pers Soc Psychol 1988;54:10631070 . 